\title{
TUCKER-KY FAN COLORINGS
}

\author{
K. S. SARKARIA
}

(Communicated by Frederick R. Cohen)

\begin{abstract}
The existence of a continuous $\mathbf{Z}_{2}$-map from a free $m$-dimensional $\mathrm{Z}_{2}$-simplicial complex $E$ to the $(m-1)$-dimensional antipodal sphere $S^{m-1}$ is characterized by means of an enumerative combinatorial criterion involving a coloring of the vertices of $E$. The Borsuk-Ulam theorem, 1933, and the combinatorial lemmas of Tucker, 1945, and Ky Fan, 1952, are easy consequences of this result for the case $|E|=S^{m}$.
\end{abstract}

\section{INTRODUCTION}

Let $E$ be a free $\mathbf{Z}_{2}$-simplicial complex equipped with a Tucker-Ky Fan coloring, i.e. a coloring of its vertices by $\{ \pm 1, \pm 2, \ldots\}$ which assigns antipodal (resp., nonantipodal) integers to antipodal (resp., contiguous) vertices of $E$. An oriented $m$-simplex of such an $E$ is called alternating iff its coloring is of the type

$$
\left[+n_{0},-n_{1}, \ldots,(-1)^{m} n_{m}\right], \quad 0<n_{0}<n_{1}<\cdots<n_{m} .
$$

For the case when $E$ is a $\mathbf{Z}_{2}$-triangulation of the antipodal $m$-sphere $S^{m}, \mathrm{Ky}$ Fan [4] showed that $E$ must have an odd number of alternating $m$-simplices. In particular, for such an $E$, a coloring of the above type must use at least $m+1$ pairs of integers. This earlier result of Tucker [12] in turn implies the Borsuk-Ulam theorem [1], viz., that there does not exist a continuous $Z_{2}$-map from $S^{m}$ to $S^{m-1}$.

The object of this note is to establish the following.

1.2. Generalized Tucker-Ky Fan theorem. Let $E$ be any free m-dimensional $\mathbf{Z}_{2}$-simplicial complex equipped with a Tucker-Ky Fan coloring. Then, there exists a continuous $\mathbf{Z}_{2}-$ map $E \rightarrow S^{m-1}$ iff the algebraical number of alternating $m$-simplices contained in any integral $\mathbf{Z}_{2}$-chain of $E$ is divisible by $2^{k}, k \geq 1$, whenever the boundary of the chain is divisible by $2^{k}$.

Here, an integral $\mathbf{Z}_{2}$-chain $c$ means an integral linear combination $\sum_{\sigma \in E} c_{\sigma} \sigma$, each simplex $\sigma$ being oriented with $-\sigma$ interpreted as the oppo-

Received by the editors September 25, 1988 and, in revised form, March 21, 1989.

1980 Mathematics Subject Classification (1985 Revision). Primary 55M35, 52A37. 
sitely oriented simplex; further $c$ is required to be symmetric (resp., antisymmetric) in even (resp., odd) dimensions with respect to the $\mathbf{Z}_{2}$-action of $E$. The oriented simplex $\sigma$ (resp., $-\sigma$ ) is said to be contained $c_{\sigma}$ (resp., $-c_{\sigma}$ ) times in the chain $c$. Finally, as usual, the boundary $\partial c=\sum_{\sigma} c_{\sigma} \partial \sigma$, the boundary of an oriented simplex being defined by

$$
\partial\left[v_{0}, v_{1}, \ldots, v_{m}\right]=\sum_{0 \leq i \leq m}(-1)^{i}\left[v_{0}, \ldots, \hat{v}_{i}, \ldots, v_{m}\right],
$$

where ' denotes omission.

Consider the case when $E$ is a minimal (w.r.t. $\subseteq$ ) $m$-dimensional pseudomanifold, i.e. each $(m-1)$-simplex is incident to two $m$-simplices. Now the boundary of an $m$-chain is divisible by $2^{k}, k \geq 1$, only if, $\bmod 2^{k}$, it is a multiple of a sum of all the $m$-simplices $\sum \pm \sigma^{m}$. Further, note that if $\sum \pm \sigma^{m}$ is such a $\mathbf{Z}_{2}$-chain, then its boundary is divisible by 2 , but is divisible by a higher power of 2 only if it is zero, and so only if $E$ is $\mathbf{Z}_{2}$-orientable, i.e. orientable with orientation-cycle symmetric (resp., antisymmetric) for $m$ even (resp., odd). So we have the following.

1.3. Corollary for $\mathbf{Z}_{2}$-pseudomanifolds. Let $E$ be a minimal m-dimensional pseudomanifold which is equipped with a free $\mathbf{Z}_{2}$-action and a Tucker-Ky Fan coloring. When $E$ is not $\mathbf{Z}_{2}$-orientable (resp., $\mathbf{Z}_{2}$-orientable) then there exists a continuous $\mathbf{Z}_{2}$-map $E \rightarrow S^{m-1}$ iff the number (resp., the algebraic number w.r.t. an orientation) of alternating $m$-simplices is even (resp., zero).

1.4. Remarks. (a) Note that $S^{m}$ is not $\mathbf{Z}_{2}$-orientable. So, the Borsuk-Ulam theorem, and the "if" part of Corollary 1.3, imply the aforementioned results by Ky Fan and Tucker. On the other hand, the octahedral $m$-sphere, $E=$ $\{ \pm 1\} \cdot\{ \pm 2\} \cdots \cdots\{ \pm(m+1)\}$-i.e. the $(m+1)$-fold join of 2 points-contains only one alternating $m$-simplex, $[+1,-2,+3, \ldots]$, under the identity Tucker-Ky Fan coloring. So the "only if" part of 1.3 also yields the Borsuk-Ulam theorem.

(b) An octahedral sphere is a particular case of a more general construction. If the vertices of a simplicial complex $K$ are named $1,2, \ldots$, then its deleted join $K_{*}$ (i.e. the simplicial complex consisting of all simplices of the type $\sigma \cup(-\theta)$, $\sigma \cap \theta=\phi$, and equipped with the free $\mathbf{Z}_{2}$-action $\left.\sigma \cup(-\theta) \mapsto \theta \cup(-\sigma)\right)$ gets the identity Tucker-Ky Fan coloring and 1.2 and 1.3 give combinatorial criteria for the existence of a continuous $\mathbf{Z}_{2}$-map from $K_{*}$ to $S^{m-1}$.

(c) It is easily checked that out of the deleted joins $\left(\sigma_{t}^{i}\right)_{*}$, of the $t$-skeleta $\sigma_{t}^{i}$ of $i$-simplices $\sigma^{i}$, the only ones which are pseudomanifolds are the octahedral $t$-spheres $\left(\sigma_{t}^{t}\right)_{*}$, which have just one alternating $t$-simplex, and the Flores $(2 t+1)$-spheres $\left(\sigma_{t}^{2 t+2}\right)_{*}$, which have $2 t+3$ alternating $(2 t+1)$-simplices. (The fact that $\left(\sigma_{t}^{2 t+2}\right)_{*}$ is $\mathbf{Z}_{2}$-homeomorphic to $S^{2 t+1}$ is not obvious and is due to Flores [3].)

(d) For $K$ to embed in $\mathbf{R}^{m-1}$ it is necessary that there be a continuous $\mathbf{Z}_{2}$ map from $K_{*}$ to $S^{m-1}$. (Much less obvious is the fact, due to C. Weber, that 
even the converse is true if $2 m-5 \geq 3 \cdot \operatorname{dim} K$.) So, by using (c) and 1.3 , it follows that the $t$-skeleton of a $(2 t+2)$-simplex-or, for that matter, the join of any number of such complexes-does not embed in a Euclidean space of twice its dimension. This result is due to van Kampen [14] and Flores [3], and the proof just given is close in spirit to the original one of van Kampen. (Flores used the Borsuk-Ulam theorem and the fact that the deleted joins of such complexes are spheres. See also [9] where it is shown that such $n$-complexes are the only ones whose deleted joins are $(2 n+1)$-pseudomanifolds.)

Our proof of Theorem 1.2 is essentially an exposition of known facts:

Following Shapiro [10] and Wu [16]-who consider "deleted products" only -we will see in $\S 2$ that the $m$ th characteristic or Smith class of $E^{m}$ can be defined in terms of its alternating $m$-simplices. Using this the enumerative combinatorial criterion of 1.2 turns out to be equivalent to saying that this class is zero. To complete the proof we now recall (this fact is probably due to $\mathrm{H}$. Hopf and was well known by 1945) why the vanishing of this class is equivalent to the existence of a continuous $\mathbf{Z}_{2}$-map $E^{m} \rightarrow S^{m-1}$.

\section{Characteristic Classes}

The underlying ring of coefficients of our (co)chains will always be $I_{(k)}$, the ring of integers $\bmod 2^{k}, 1 \leq k \leq \infty$ (note that $I_{(\infty)}=I$, the ring of integers). We will consider two actions of the 2-letter group $\mathbf{Z}_{2}=\{\mathrm{Id}, \nu\}$ on this ring: the trivial or symmetric action $\nu(x)=+x$, and the antisymmetric action $\nu(x)=-x$. A (co)chain of $E$ will be called equivariant (i.e. symmetric or antisymmetric) if it is preserved by the action obtained by combining \pm with the given $\mathrm{Z}_{2}$ action of $E$.

We note that the notion of a Tucker-Ky Fan coloring of $E$ is obviously equivalent to that of a simplicial $\mathrm{Z}_{2}$-map $\varphi$ from $E$ to the infinite dimensional, or universal octahedral sphere $U=\{ \pm 1\} \cdot\{ \pm 2\} \cdots$ (i.e. the infinite join $\mathbf{Z}_{2} \cdot \mathbf{Z}_{2} \cdots$ of the group under consideration). The least number of pairs of integers required to color $E$ will be called its combinatorial genus $c \cdot g \cdot(E)$. Clearly every free $\mathbf{Z}_{2}$-complex $E$ does have a Tucker-Ky Fan coloring and $c \cdot g \cdot(E) \leq N$, where $N$ is the number of pairs of vertices of $E$.

The equivariant cohomology $H_{ \pm}^{*}(U)$ of the universal octahedral sphere is also called the cohomology of the group $\mathbf{Z}_{2}$ and denoted by $H_{ \pm}^{*}\left(\mathbf{Z}_{2}\right)$. Next, note that any two Tucker-Ky Fan colorings $\varphi_{1}, \varphi_{2}: E \rightarrow U$ are $\mathbf{Z}_{2}$-homotopic: to see this translate $\varphi_{1}$ to $\varphi_{1}^{\prime}$ so that $\varphi_{1}^{\prime}, \varphi_{2}$ employ disjoint sets of colors, and then join $\varphi_{1}^{\prime}$ to $\varphi_{2}$ linearly. So the (characteristic) homomorphism $\varphi^{*}: H_{ \pm}^{*}\left(\mathbf{Z}_{2}\right) \rightarrow H_{ \pm}^{*}(E)$ defined by means of a Tucker-Ky Fan coloring $\varphi$ of $E$ is in fact independent of $\varphi$. The equivariant cohomology classes of $E$ lying in its image are called the characteristic classes of $E$.

In particular, for $i$ even (resp., odd) let $a_{i}$ denote the symmetric (resp., antisymmetric) cochain of $U$ which has the values 1,1 (resp., 1, -1) on alternating $i$-simplices and their antipodes, and the value 0 on all other sim- 
plices. From 2.1 below it follows that $a_{i}$ is in fact a cocycle, i.e. $\delta a_{i}=0$; thus we have the characteristic classes $\left[\varphi^{*} a_{i}\right] \in H_{ \pm}^{i}\left(E ; I_{(k)}\right), 1 \leq k \leq \infty$.

2.1. Coboundary formula. Let $\bar{a}_{i}$ denote the cochain of $U$ which takes the value 1 on alternating simplices and the value 0 on all other simplices. Then $\delta\left(\bar{a}_{i}\right)=(-1)^{i+1} a_{i+1}$ for all $i>0$.

Proof. Alternating $(i+1)$-simplices-see (1.1)-or their antipodes do occur in $\delta \bar{a}_{i}$ because they are incident to precisely one alternating $i$-simplex: the one obtained by omitting the last or the first vertex, respectively. The only other $(i+1)$-simplices which could have occurred are those whose vertices, when totally ordered by absolute value, alternate in sign with just one exception: but now one has two alternating $i$-faces occurring with opposite incidence numbers.

Thus $\varphi^{*} a_{i+1}$ is the coboundary of an ordinary cochain, however it need not be the coboundary of an equivariant cochain. In fact

2.2. The combinatorial criterion given in 1.2 is equivalent to saying that the characteristic class $\left[\varphi^{*} a_{m}\right] \in H_{ \pm}^{m}\left(E ; I_{(k)}\right)$ is zero for all $1 \leq k<\infty$.

Proof. For any equivariant cochain $a$, and chain $c=\sum_{\sigma \in E} c_{\sigma} \sigma$, define $\langle a, c\rangle=\sum_{\sigma \in E / \mathbf{Z}_{2}} c_{\sigma} a(\sigma)$, where in the summation only one representative is chosen from each orbit $\{\sigma, \nu(\sigma)\}$. Note that $\left\langle\varphi^{*} a_{m}, c\right\rangle$ equals the algebraical number of alternating $m$-simplices contained in $c$. Also, that the $I_{(k)}$-modules of equivariant cochains and chains are dual to each other under this unimodular bilinear form $\langle$,$\rangle , and one has Stokes formula \langle\delta a, c\rangle=\langle a, \partial c\rangle$. So if $\varphi^{*} a_{m}$ is the coboundary of an equivariant cochain, and $c$ is any equivariant cycle $\bmod 2^{k}$, then one must have $\left\langle\varphi^{*} a_{m}, c\right\rangle=0 \bmod 2^{k}$.

Conversely, if $\varphi^{*} a_{m}$ is not in $\operatorname{Im} \delta$, then let $r$ be the smallest positive integer such that $r \varphi^{*} a_{m} \in \operatorname{Im} \delta$. Note that $r$ divides $2^{k}$, i.e. $r=2^{l}$ for some $1 \leq l \leq k<\infty$. Let $c$ be an equivariant chain for which the corresponding $I_{(k)}{ }^{-}$ valued linear function $\langle, c\rangle$ is zero on $\operatorname{Im} \delta$ and takes the (nonzero $\bmod 2^{k}$ ) value $2^{k-l}$ on $\varphi^{*} a_{m}$. This $c$ is a $\bmod 2^{k}$ cycle because $\langle a, \partial c\rangle \in I_{(k)}$ is zero for all $a$.

We now check the "only if" part of 1.2. The least $t$ such that there exists a continuous $\mathrm{Z}_{2}$-map from $E$ into $S^{t-1}$ will be called the topological genus $t \cdot g \cdot(E)$ of $E$; note that $t \cdot g \cdot(E) \leq c \cdot g \cdot(E)$.

2.3. $T \cdot g \cdot(E) \leq \operatorname{dim}(E)+1$ and all characteristic classes of $E$ vanish in dimensions $\geq t \cdot g \cdot(E)$.

Proof. Let $E^{\prime}$ denote the $\mathbf{Z}_{2}$-simplicial complex whose simplices are chains (under $\subseteq$ ) of nonempty simplices of $E$, i.e. the simplicial complex obtained by performing a barycentric subdivision of $E$. One has $t \cdot g \cdot(E)=t \cdot g \cdot\left(E^{\prime}\right) \leq$ $c \cdot g \cdot\left(E^{\prime}\right)=\operatorname{dim}(E)+1$, because $E^{\prime}$ has Tucker-Ky Fan colorings using just $\operatorname{dim}(E)+1$ colors, viz., those in which $i$-dimensional barycenters are colored $\pm(i+1)$. 
Further, simplicial approximation shows that there exists a continuous $\mathbf{Z}_{2}$ map $\left.E \stackrel{f}{\longrightarrow} S^{t-1}=\{ \pm 1\} \cdots \cdot \pm t\right\}$ iff there exists a simplicial $\mathbf{Z}_{2}$-map $\bar{f}$ from $E^{(r)}$, a sufficiently fine barycentric subdivision of $E$, into $\left.\{ \pm 1\} \cdots, \pm t\right\} \subset$ $U$. Since $\bar{f}^{*} a=0$ for all cochains $a$ of $U$ of dimension $\geq t$, it would suffice thus to check that the characteristic homomorphisms of $E^{(r)}$ and $E$ are related to each other by the isomorphism $H_{ \pm}^{*}\left(E^{(r)}\right) \stackrel{\cong}{\longrightarrow} H_{ \pm}^{*}(E)$ induced by chain subdivision. This is indeed so because the inverse isomorphism can be induced from a $\mathrm{Z}_{2}$-simplicial map $E^{(r)} \rightarrow E$, viz., one which maps each vertex of $E^{(r)}$ to some vertex of the open simplex of $E$ containing it.

Since integral coefficients are needed for the "if" part of 1.2 we first verify the following.

2.4. The combinatorial criterion given in 1.2 is equivalent to saying that the integral characteristic class $\left[\varphi^{*} a_{m}\right] \in H_{ \pm}^{m}(E ; I)$ is zero.

Proof. We note first that the characteristic class is of order 2: this follows e.g. from the coboundary formula 2.1 which shows that $2 a_{m}$ is the coboundary of the equivariant cochain $u_{m-1}=(-1)^{m}\left(\bar{a}_{m-1}+(-1)^{m} \nu^{*}\left(\bar{a}_{m-1}\right)\right)$. Now suppose that $\varphi^{*} a_{m}$ is an equivariant coboundary $\bmod 2^{k}$, i.e. $\varphi^{*} a_{m}=\delta v+2^{k} w$ for some equivariant cochains $v$ and $w$. So $\delta w=0$ and $2^{k+1} w$ is the coboundary of the equivariant cochain $\varphi^{*} u_{m-1}-2 v$. If $k$ is so large that $H_{ \pm}^{m}(E ; I)$ has no element of order $2^{k+1}$, we must have $w=\delta x$ for some equivariant $x$. So $\varphi^{*} a_{m}=\delta\left(v+2^{k} x\right)$ and $\left[\varphi^{*} a_{m}\right] \in H_{ \pm}^{m}(E ; I)$ is zero. Since, conversely, $\varphi^{*} a_{m}$ can be an equivariant coboundary only if it is so $\bmod 2^{k}$ for all $1 \leq k<\infty$, the result follows by using 2.2 .

So the next result completes the proof of Theorem 1.2.

2.5. If the highest-dimensional integral characteristic class $\left[\varphi^{*} a_{m}\right] \in H_{ \pm}^{m}\left(E^{m} ; I\right)$ is zero, then there exists a continuous $\mathbf{Z}_{2}$-map $f: E^{m} \rightarrow S^{m-1}$.

Proof. We equip $\mathbf{R}^{m}$ with the antipodal $\mathbf{Z}_{2}$-action $\nu(x)=-x$ (having the unique fixed point $O$ ) and use the notation $[t]$ for the point $\left(t, t^{2}, \ldots, t^{m}\right)$ of the moment curve of $\mathbf{R}^{m}$. Let $\mu_{m}: U \rightarrow \mathbf{R}^{m}$ denote the linear $\mathbf{Z}_{2}$-map which images the vertices $\pm 1, \pm 2, \ldots$, of the universal octahedral sphere $U$ to the points $\pm[1], \pm[2], \ldots$, of $\mathbf{R}^{m}$. Note that $\mu_{m}$ is $1-1$ on simplicies $\sigma$ of $U$ of dimensions $\leq m$. Further, one can verify that the image $\mu_{m}(\sigma)$ of such a simplex contains $O \in \mathbf{R}^{m}$ iff $\sigma$ or $\nu(\sigma)$ is an alternating $m$-simplex of $U$.

The composition $\mu_{m} \varphi$ provides us with a continuous $\mathbf{Z}_{2}$-map from the $(m-1)$-skeleton $E^{m-1}$ to $\mathbf{R}^{m} \backslash\{O\}$, and we can replace this by a homotopic $\mathbf{Z}_{2}$-map $g: E^{m-1} \rightarrow S^{m-1}$. The cochain $\varphi^{*} a_{m}$ takes the values 1 and $(-1)^{m}$ on alternating $m$-simplices of $E$ and their antipodes, and the value 0 on all other simplices. Thus, with an appropriate orientation of $S^{m-1}, \varphi^{*} a_{m}\left(\sigma^{m}\right)$ 
coincides with the degree of the restricted map $g \mid \partial \sigma^{m}: \partial \sigma^{m} \rightarrow S^{m-1}$ for all $\sigma^{m} \in E$.

The required $Z_{2}$-map can now be found by the following well-known procedure. Choose an equivariant $(m-1)$-cochain $u$ such that $\delta u=\varphi^{*} a_{m}$. Replace $g$ by a continuous $\mathbf{Z}_{2}$-map $f: E^{m-1} \rightarrow S^{m-1}$ which is equal to $g$ on $E^{m-2}$, and differs from $g$, on each $\theta^{m-1} \in E$, by the degree amount $u\left(\theta^{m-1}\right)$. By virtue of $\delta u=\varphi^{*} a_{m}$ each restriction $f \mid \partial \sigma^{m}, \partial \sigma^{m} \in E$, has degree 0 . So we can extend this map to the required continuous $\mathbf{Z}_{2}$-map $f: E^{m} \rightarrow S^{m-1}$.

\section{Notes}

3.1. Deleted joins are maximal free $\mathbf{Z}_{2}$-simplicial complexes in the sense that a $1-1$ Tucker-Ky Fan coloring $\varphi$ of any $E$ exhibits it as a $\mathbf{Z}_{2}$-subcomplex of $K_{*}$, where $K={ }^{\varphi} E$, the full subcomplex of $E$ determined by the positive vertices. Furthermore if $E$ is a deleted join, then one can verify that ${ }^{\varphi} E$ is independent of $\varphi$ up to simplicial isomorphism. This reconstruction property is not shared by the more common "deleted products" $K_{\#}$ (the sub cell complex of $K \times K$ consisting of all cells $\sigma \times \theta, \sigma \cap \theta=\phi)$ e.g. $\sigma_{n}^{n}$ and $\sigma_{n-1}^{n}$ have the same deleted product. However an affirmative solution of the well-known graph reconstruction problem of Ulam [13] (p. 29), would show that nonisomorphic graphs having more than three vertices cannot have $Z_{2}$-isomorphic deleted products.

Regarding deleted joins note also that if $K$ has $N$ vertices, then $K_{*}$ must have combinatorial genus $>N / 3$ : a lesser number of colors would result in some three vertices of $K_{*}$ getting the same color and the coloring would be bad on the hexagon $\left(\sigma_{0}^{2}\right)_{*} \subseteq K_{*}$ determined by these vertices and their antipodes.

3.2. The proof of 2.5 amounted to checking that $\varphi^{*} a_{m}$ is the obstruction-see [11]-experienced in trying to extend a continuous $\mathrm{Z}_{2}$-map $g: E^{m-1} \rightarrow S^{m-1}$ to all of $E^{m}$. For $E=K_{*}$ this is equivalent to the definition of van Kampen [14], who considered cocycles which count the algebraical number of isolated self-intersections of a general position map $h: K \rightarrow \mathbf{R}^{m-1}$ e.g., (see [10]) the linear map $h(i)=[i]$ yields the alternating cocycle $\varphi^{*} a_{m}$.

The universal space definitions of characteristic classes (see [11] and [5]) came later. Still another approach (see [17]) is to note that the equivariant cochain subcomplexes $C_{ \pm}^{*}(E)$ of $C^{*}(E)$ fit into the two short exact sequences

$$
0 \rightarrow C_{ \pm}^{*}(E) \hookrightarrow C^{*}(E) \stackrel{\mathrm{Id} \mp \nu^{*}}{\longrightarrow} C_{\mp}^{*}(E) \rightarrow 0
$$

and so give rise to the following two long exact sequences

$$
\cdots \rightarrow H_{ \pm}^{i}(E) \rightarrow H^{i}(E) \rightarrow H_{\mp}^{i}(E) \stackrel{\delta_{ \pm}}{\longrightarrow} H_{ \pm}^{i+1}(E) \rightarrow \cdots
$$

of Richardson and Smith [6]. By repeatedly applying the connecting homomorphisms $\delta_{ \pm}$to the unit class [1] $\in H_{+}^{0}(E)$ one obtains the Smith classes of $E$. 
The coboundary formula 2.1 shows that these coincide up to sign with the alternating classes $\left[\varphi^{*} a_{m}\right]$. With mod 2 coefficients there is only one long exact sequence and its exactness shows immediately (cf. [15]) that if $E$ is homologically trivial in dimensions less than $m$, then $\left[\varphi^{*} a_{m}\right]$ is nonzero. For $E=K_{*}$ this approach relates these Smith, or Stiefel-Whitney, classes to the cohomology operations of $K$.

We will show elsewhere (see also $[2,7,8]$ ) that one can obtain a similar combinatorial understanding of other characteristic classes by generalizing 1.2 to free actions of finite groups $G$ other than $\mathbf{Z}_{2}$.

\section{REFERENCES}

1. K. Borsuk, Drei Sätze über die n-dimensionale euklidische Sphäre, Fund. Math. 20 (1933), 177-190.

2. A. Dold, Simple proofs of some Borsuk-Ulam results, Contemp. Math. 19 (1983), 65-69.

3. A. Flores, Über n-dimensionale Komplexe, die im $R_{2 n+1}$ absolut selbstverschlungen sind, Ergeb. math. Kolloq. 6 (1933/34), 4-7.

4. Ky Fan, A generalization of Tucker's combinatorial lemma with topological applications, Ann. of Math. 56 (1952), 431-437.

5. J. W. Milnor, Construction of universal bundles I, II, Ann. of Math. (2) 63 (1956), 272-284, 430-436.

6. M. Richardson and P. A. Smith, Periodic transformations of complexes, Ann. of Math. (2) 39 (1938), 611-633.

7. K. S. Sarkaria, A generalized Kneser conjecture, J. Combin. Theory Ser. B (to appear).

8. __ A generalized van Kampen-Flores theorem, Proc. Amer. Math. Soc. (to appear).

9. __ Kuratowski complexes, Topology (to appear).

10. A. Shapiro, Obstructions to the imbedding of a complex in a Euclidean space. I. The first obstruction, Ann. of Math. (2) 66 (1957), 256-269.

11. N. E. Steenrod, The topology of fibre bundles, Princeton Univ. Press, Princeton, 1951.

12. A. W. Tucker, Some topological properties of disk and sphere, in Proceedings of the First Canad. Math. Congress, 1945, pp. 285-309.

13. S. M. Ulam, A collection of mathematical problems, Wiley, New York, 1960.

14. E. R. van Kampen, Komplexe in euklidischen Räumen and Berichtigung, Abh. Math. Sem. Univ. Hamburg 9 (1932), 72-78 and 152-153.

15. J. W. Walker, A homology version of the Borsuk-Ulam theorem, Amer. Math. Monthly 90 (1983), 465-468.

16. W.-T. Wu, On the realization of complexes in Euclidean spaces I, Acta Math. Sinica (N.S.) 5 (1955), 505-552; Sci. Sinica 7 (1958), 251-297.

17. _ _ A theory of imbedding, immersion, and isotopy of polytopes in a Euclidean space, Science Press, Peking, 1965.

Department of Mathematics, Panjab University, Chandigarh 160014, India 